\title{
Experimental investigation of water droplets' behavior in dielectric medium: the effect of an applied D.C. electric field
}

\author{
H. Bararnia and D. D. Ganji \\ Department of Mechanical Engineering, Babol University of Technology, Babol, Iran \\ Correspondence to: H. Bararnia (hasan_bararnia@yahoo.com)
}

Received: 27 July 2013 - Revised: 14 September 2013 - Accepted: 27 September 2013 - Published: 11 October 2013

\begin{abstract}
In this article, the behavior of water droplets which are suspended in silicon oil is qualitatively investigated and some phenomena such as liquid's burst are reported. The movement of droplet in perpendicular line is considered while many studies have considered small droplets fluctuating between two horizontal electrodes. Additionally any deformation caused by increasing voltage was observed from start to finish until short contact occurred. As can be seen two oppositely charged drops contacting each other do not necessarily result in coalescence. Repelling can be expected occasionally. By increasing the voltage, the droplets, which spread through the domain due to frequent breakups, tend to gather in a certain line.
\end{abstract}

\section{Introduction}

Understanding the interaction of suspending conducting liquids with the second dielectric medium is a prominent factor in many engineering projects. One of the most important examples is emulsification. Regarding modifying the properties of the polymer, antistatic agents are added during mixing processes. Dispersion also enhances the mass transfer operations in liquid-liquid extraction. In oil industries, determining the onset and type of deformation is an important matter because the breakup of a drop into smaller droplets is a Preventive element concerning electrocoalescers. Nevertheless this separation could be utilized beneficially relating to emulsification and enhancing heat and mass transfer. Traditionally the tiny droplet gathering in continuous oil phase has attracted great attention in oil recovery technology; see Eow et al. (2001). Controlling the fusion of individual droplets in digital micro fluidic applications were investigated recently by Ahn et al. (2006). Applications, has been shown in some other aspects such as electro spraying by Bailey (1999) and nucleate boiling by Dong et al. (2006). Flows generated by electroosmosis have been used to act as a machine and enrich the mixing of substances at the micro level; see Pikal (2001). Dielectrophoresis have been used widely in separation for bio-cells and carbon nanotubes; see Aldaeus at al. (2006).
Considering the suspension of a droplet in immiscible medium, the shear and electrical stresses caused by external electric field and distinction electrical characteristic at the interface is expected. This results in burst of the droplet or elongation. Initially assuming the droplet as ideal conductor or fully insulator resulted no fluid flow because of inequality of shear stresses. Furthermore, based on electrostatic theory the consequent stresses are perpendicular to the surface and towards the fluid with lower permittivity. The deformation led the droplet to be prolate. The experiment conducted by Allan and Mason (1962) showed that conducting droplet deforms into prolate spheroid compatible with the electrostatic theory. They have considered several fluid systems and declared some droplets get oblate shape. Taylor (1966) pointed out considering drop as perfect conducting or dielectric fluid could not be proper in all situations. They stated that limited amount of permittivity or conductivity allows unbounded charge to collect at the droplet surface and this collection is responsible for inequality in shear and normal electrical stresses which causes oblate shape. This theory is named leaky-dielectric theory. As illustrated by Taylor, Fluid flow in the inner and outer part of the droplet is caused by balancing the tangential stresses by hydrodynamic tangential stresses. Taylor justified Allan and Mason results 


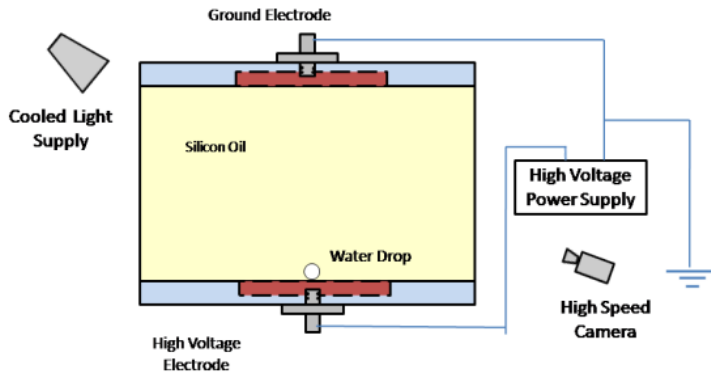

(a)

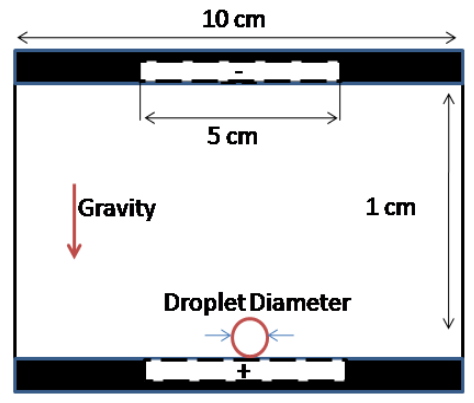

(b)

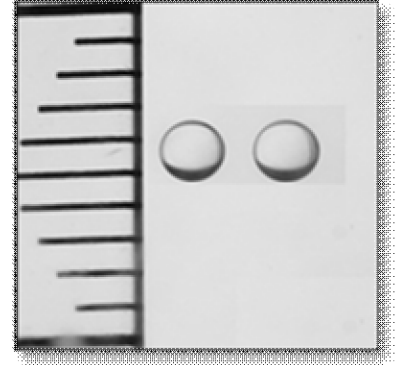

(c)

Figure 1. (a) Experimental apparatus, (b) the geometric of the cell, (c) measuring droplet's diameter.

by solving electro hydrodynamic's equation in creeping flow regime. Torza et al. (1971) revealed some discrepancies between theory and experiments. Baygents and Saville (1989) replaced leaky dielectric theory by an electrokinetic model to inspect the matters proposed by Torza et al. (1971). Feng and Scott (1966), Vizika and Saville (1992) have demonstrated that the leaky dielectric model has the capability to predict the deformation pattern while zero amounts of charges accumulated at the interface.

The research into the stabilization and breakup of an aqueous droplet is still continuing and in this section we aim to concentrate on some recent and pertinent ones. Sherwood (1988) used boundary integral method for droplets with different set of electrical properties to investigate the droplet deformation which is exposed to an electric field until burst of droplets. Ha and Yang (2000) experimented on Newtonian and non-Newtonian droplets surrounded by dielectric medium. They reported that when either the dispersed or continuous phase is non-Newtonian the droplet will be stable in low electric field. Besides, more complex behaviors are caused when both the mediums are non-Newtonian due to the zero-shear-rate viscosities ratio. Ristenpart et al. (2009) experimentally showed that there is a certain value of electric field which leads the two droplets with different sign to repel each other at the contact time while coalescence was expected. Breakup and deformation of aqueous drops in oil was investigated experimentally by Eow and Ghadiri (2003a). The results indicated that the starting point of fluctuation related to the deformation rate is about 1.9. Eow and Ghadiri (2003b) also investigated experimentally drop-drop coalescence. Their results indicated that coalescence depends on electrode geometry and orientation of the field. To achieve the maximum attractive value it is necessary to direct the center line of the droplets in line with the electric field.

Hase et al. (2006) performed experimental test to find micro-droplet's behavior which is rhythmically moved between two horizontal electrodes in silicon oil. Depending on applied electric field, three distinct zones were charac- terized. Chiesa et al. (2005) provided the analytical formula to demonstrate the forces induced by an electric field on a falling droplet. Their results were well-matched to experimental outputs. Khorshidi et al. (2010) investigated experimentally the water droplet's motion and shape which was large in size and also calculated numerically the amounts of accumulated charges as a consequence of contacting with the electrodes. They reported that neutral water droplet tends to move toward the positive electrode and named this action as negative-electrophoresis. Recently Hokmabad et al. (2012) investigated experimentally the motion and disintegration of droplets gathering charges while leaving the nozzles. They have pointed out that more deformation is concluded by increasing the voltage and the maximum value has been depicted near the nozzles. Discharging of droplets to the negative voltage (ground) leads to two distinguished modes of drop's burst. In this report, some important aspects of water droplets' behavior in a high voltage electric field are investigated. (i) electrical charging and rhythmic motion, (ii) drop-drop coalescence and the effect of existed torque, and (iii) different breakup phenomenon and breaking the oil. All sections are examined by increasing the electric field; this is the only parameter that influences the droplets' behavior.

\section{Experimental set up}

The experimental cell, which is shown in Fig. 1, consisted of four Plexiglas walls (1 mm thickness) and was filled with silicone oil as the dielectric continuous phase. Table 1 gives the relevant properties of the employed fluids. The static electric field $\left(0<\boldsymbol{E}<12 \mathrm{kv} \mathrm{cm}^{-1}\right)$ was formed between two parallel electrodes $\left[5 \times 5 \mathrm{~cm}^{2}\right.$ stainless steel plate $]$ and spaced $1 \mathrm{~cm}$ apart. The top electrode was electrically grounded, and the bottom electrode, which was connected to a D.C. power supply, acted as the high voltage electrode.

To start the experiment, two deionized water drops of $1.7 \mathrm{~mm}$ were released into the stationary dielectric oil using a syringe pump. Before applying the electric potential, the drops were initially $7.6 \mathrm{~mm}$ apart and, resting on the bottom 
Table 1. Physical properties of employed liquids.

\begin{tabular}{llll}
\hline Property & Silicon oil & Water & $\begin{array}{l}\text { Ratio } \\
\text { (oil/water) }\end{array}$ \\
\hline Viscosity $\left(\mathrm{kg} \mathrm{ms}^{-1}\right)$ & $9.6 \mathrm{e}-2$ & $1.12 \mathrm{e}-3$ & 85.7142 \\
Density $\left(\mathrm{kg} \mathrm{m}^{-3}\right)$ & 963 & 998.2 & 0.9647 \\
Conductivity $\left(\mathrm{S} \mathrm{m}^{-1}\right)$ & $14 \mathrm{e}-13$ & $1 \mathrm{e}-4$ & $14 \mathrm{e}-9$ \\
Relative permittivity & 2.75 & 80.1 & $3.4332 \mathrm{e}-2$ \\
Surface tension $\left(\mathrm{N} \mathrm{m}^{-1}\right)$ & 0.020 & 0.072 & 0.2777 \\
\hline
\end{tabular}

electrode. Then, the electric field strength was slowly raised to the critical value, above which the drops left the bottom plate and migrated towards the top (grounded) electrode. The complete history of the drop pair throughout the experiment was photographed by a CCD Camera (Casio EXILIM Pro EX-F1) at $300 \mathrm{fps}$.

\section{Results}

In this section, the whole process of the drops' behavior under the external electric field is discussed in detail.

\subsection{Rhythmic motion of the droplets}

The sequential images of the initial motion of the water drop pair in silicone oil are presented in Fig. 2. Drops are rest on the high voltage (i.e., the high voltage electrode), keeping their nearly spherical shape, while the applied voltage across the electrodes gradually increases. When the force exerted by electric field overcomes the gravity force (neglecting the surface tension between the liquid and solid surfaces), the drops are detached from the metal surface. Leaving the high voltage electrode, the drops acquire a positive charge and go against gravity towards the upper electrode. By contacting the grounded (upper) electrode, they exchange their charge and go back to the lower electrode, where they gather positive charge again and repeat this cycle. In the first few cycles, the drops travel simultaneously and reach the same electrode; eventually, the rhythmic motion becomes inverted in such a way that when the first drop touches an electrode, the other touches the opposite electrode. This change in direction causes the drops to become oppositely charged and attracted to each other while moving up and down in the surrounding dielectric oil.

It can be observed that the drops' velocity increases after contact with the electrode surface. As the drops' motion is entirely electrically driven, the increase in velocity magnitude reveals an increase in the accumulated charge on the drops' surfaces during their rhythmic motion.

The trend of drop position prior to their coalescence is plotted in Fig. 3a. As observed, the rise in the voltage yields to decline the frequency. Unlike the assumption that the reverse should be true (i.e., that a higher frequency is expected), the results have shown that the movement energy gained from increased voltage is consumed such that the drops migrate towards each other and, consequently, become closer. Therefore, the time needed to travel between the electrodes is longer than in the case where each drop moves in a vertical line at their initial positions. The voltage value was increased up to $3.8 \mathrm{kv} \mathrm{cm}^{-1}$ when coalescence occurred. In general, anticipating the drop behavior in a high electric field is very complex. The characteristics of employed fluids, the uniformity and the orientation of the applied electric field compared to the drop, are leading factor. Under the uniform electric field, drops become polarized while bouncing up and down and develop a conical shape oriented in the orientation of the field. As the drops come closer to the electrode, the distance between droplets and electrode is reduced, leads to higher electric force and results in the elongation of the drop. The electric body forces experienced by a spherical charged droplet can be expressed as follows:

$\boldsymbol{F} e=Q \boldsymbol{E}+(2 \pi R) \varepsilon_{1}\left(\frac{\varepsilon_{2}-\varepsilon_{1}}{\varepsilon_{2}+2 \varepsilon_{1}}\right) \nabla\left|E^{2}\right|$

where $Q$ is the free charge density. Here $\varepsilon_{1}$ and $\varepsilon_{2}$ are the continuous and dispersed phase electrical permittivity, respectively. The first term is Coulomb force (electrophoresis), and it describes the affect of the electric field on the net charge $Q$ of the drop. In a D.C. case, Coulomb force exists only in the existence of a net charge on the droplet. The second term, referred to as the dielectrophoretic force (DEF), generated by non-uniformity in the electric field and electrical properties of materials. This force only disappears if the field is spatially homogeneous. (It is worth mentioning that the drop causes the electric field to become non-uniform in its neighboring.) Generally both of magnetism and electricity are included in the basic Maxwell's equation; however, for a wide class of problems, the magnetism is decoupled in the case of weak electric current or low magnetic field. In the vicinity of the electrode, the droplet become elongated sharper and consequently contacts the electrode by making a conical tip referred to as the "Taylor cone". Subsequently, the cone recoils, and the drops reverse their direction and move toward the opposite electrode. By increasing the voltage, the deformation rate (elongation) increases and, in some cases, will result in short contact. However at low voltages, the drop tends to keep a spherical shape at the midpoint between the electrodes due to surface tension. In the middle, the weight and drag force affect the drop and restrict the motion that originated from the electric force. However, near the electrodes drops experience excessive electric stress due to the short distance and accelerate at the time of detachment from the electrodes. Moreover, each of the polarized drops experiences a dielectrophoretic attraction force since the existence of second droplet in its vicinity, which brings them towards each other. The dipole-dipole interaction force is very small when the two drops are far, and its effect can be neglected compared to the electrophoretic force. However, when the drops are nearness in distance, the dipole-dipole attraction is 

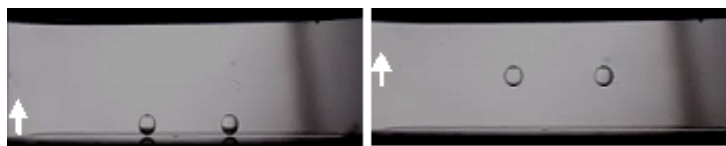

Initial position $(\mathrm{T}=0)$

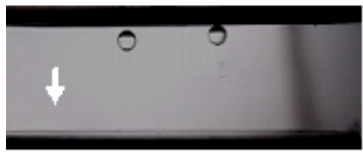

$\mathrm{T}=60.06 \mathrm{~s}$
$\mathrm{T}=40.98 \mathrm{~s}$

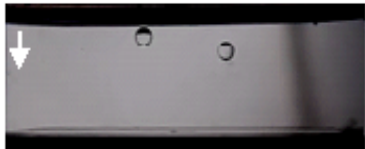

$\mathrm{T}=71.88 \mathrm{~s}$

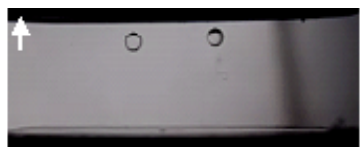

$\mathrm{T}=52.98 \mathrm{~s}$

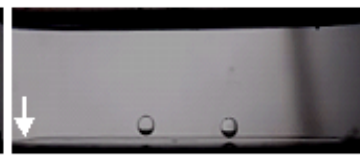

$\mathrm{T}=90.78 \mathrm{~s}$

Figure 2. Sequential images showing the motion of a pair of water drops subjected to a D.C. Electric field $\boldsymbol{E} o=1.1 \mathrm{kV} \mathrm{cm}^{-1}$ for drop radius $0.875 \mathrm{~mm}$.
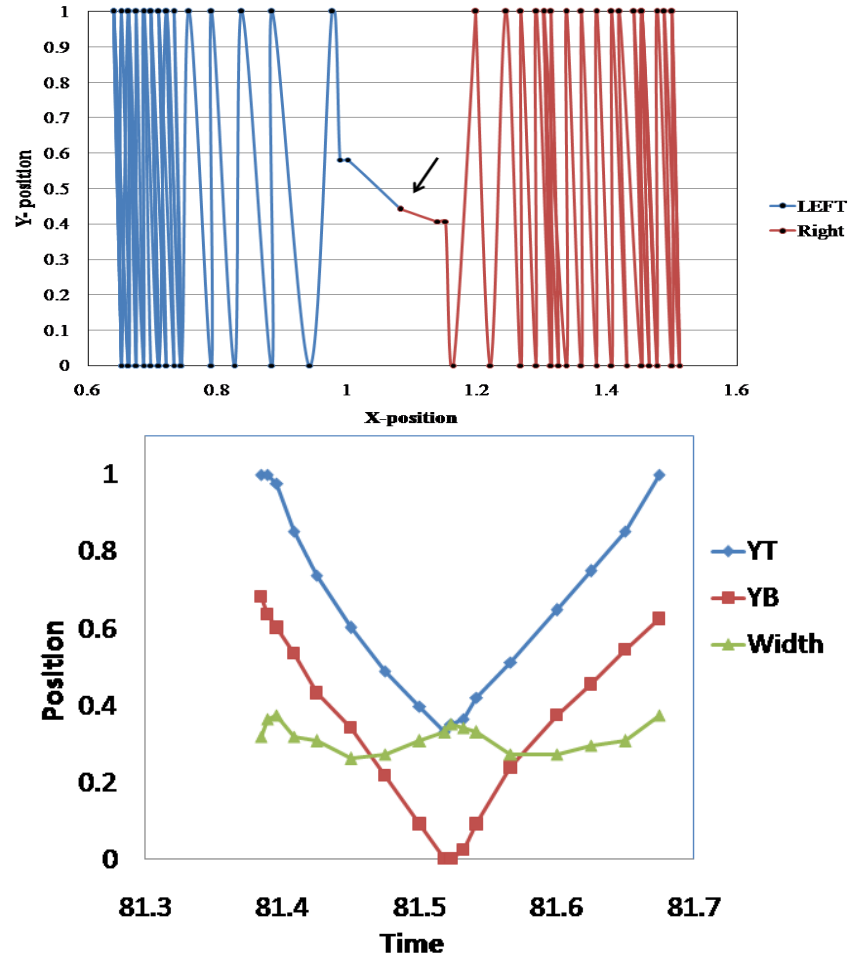

Figure 3. (a) Position of each drop versus time until coalescence, (b) Deformation of a drop in a cycle at $\mathrm{Ca}_{E}=0.018$ (Yt: the top point of droplet, $\mathrm{Yb}$ : the bottom point of droplet).

strong, which induces deformation on the drop pair and results in their coalescence.

\subsection{Two drops deformation and coalescence}

Figure 4 shows captured images where the drops move towards each other and merge into one. For two drops moving near each other, coalescence may occur due to hydrodynamical forces, however, in high electric field, electrostaticcoalescence is predominant factor. In an electro-coalescence phenomenon, when the electric force becomes high enough to push the drops towards each other, it triggers the oil film drainage between the drops and facilitates their contact, which, in most cases, results in coalescence, as illustrated in Fig. 4. When the electric field is not sufficient to develop drop-drop merging, the drops restore to their former state and keep their rhythmic motion between the electrodes.

At the time of approaching the drops, a thin layer separates the surface of the drops from the continuous phase and by rupturing the interfacial layer the drop-drop coalescence is predicted. Collectively, the roles of the electric field are; develop contact between the water drops, improve coalescence and enhance breaking the drop-interface. There are some other factors among these functions, such as the influence of electric field orientation on the coalescence of two aqueous drops, the influence of electrode geometry, which may play an important role in coalescence performance. However, it should be considered that occasionally two drops change their charges before coalescence takes place. This change can generate electrophoretic forces, which are responsible for repelling the drops from each other. One of the major factors that prevent the two dispersed drops to coalesce is drainage of the layer which separates them. By breaking this layer the chance of occurring coalescence grows rapidly. As observed, before coalescence the drops' tips face each other and deform. Furthermore, coalescence forms a dumbbell drop. There are two processes, i.e., before and after the occurring coalescence. Before coalescence, the two drops moved in a vertical direction. Considering the three dimensional behavior and distance between the two drops, it can be observed that the drops are brought closer together because of the two opposite charges, and when the distance is short enough, the torque rotates the drop while the drop pair becomes aligned in the electric field's orientation. This local rotation torque, which is due to dipole-dipole attraction, disappears when the drops become parallel and appears when they pass through each other (Fig. 5).

This torque is related to the minimum interaction energy, which depends on the distance between dipoles, permittivity of the two phases, polarization rate of the drops and direction of the induced dipole of a drop relative to the electric field orientation. Once the dipoles become far apart, the torque 


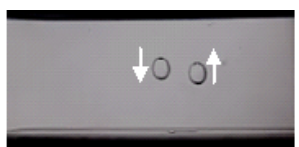

$\mathrm{T}=262.60 \mathrm{~s}$

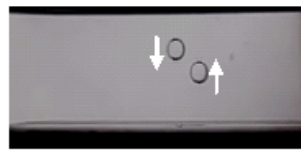

$\mathrm{T}=264.80 \mathrm{~s}$

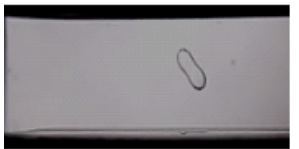

$\mathrm{T}=266.41 \mathrm{~s}$

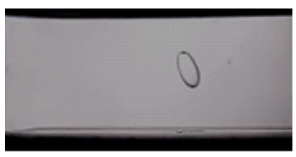

$\mathrm{T}=266.48 \mathrm{~s}$

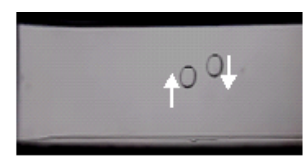

$\mathrm{T}=264.40 \mathrm{~s}$

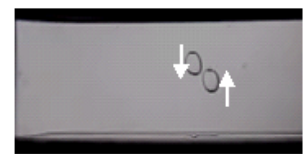

$\mathrm{T}=266.30 \mathrm{~s}$

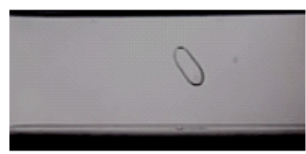

$\mathrm{T}=266.45 \mathrm{~s}$

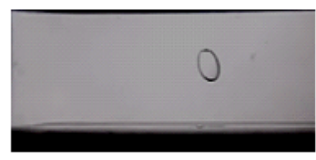

$\mathrm{T}=266.49 \mathrm{~s}$

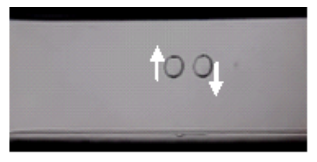

$\mathrm{T}=264.50 \mathrm{~s}$

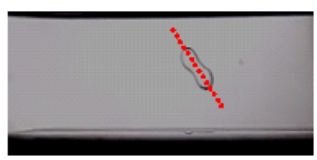

$\mathrm{T}=266.40 \mathrm{~s}$

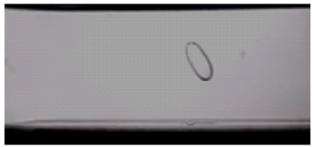

$\mathrm{T}=266.46 \mathrm{~s}$

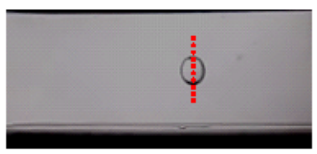

$\mathrm{T}=266.50 \mathrm{~s}$

Figure 4. Interaction leading merging between two water drops at $\mathrm{Ca}_{E}=0.18$.

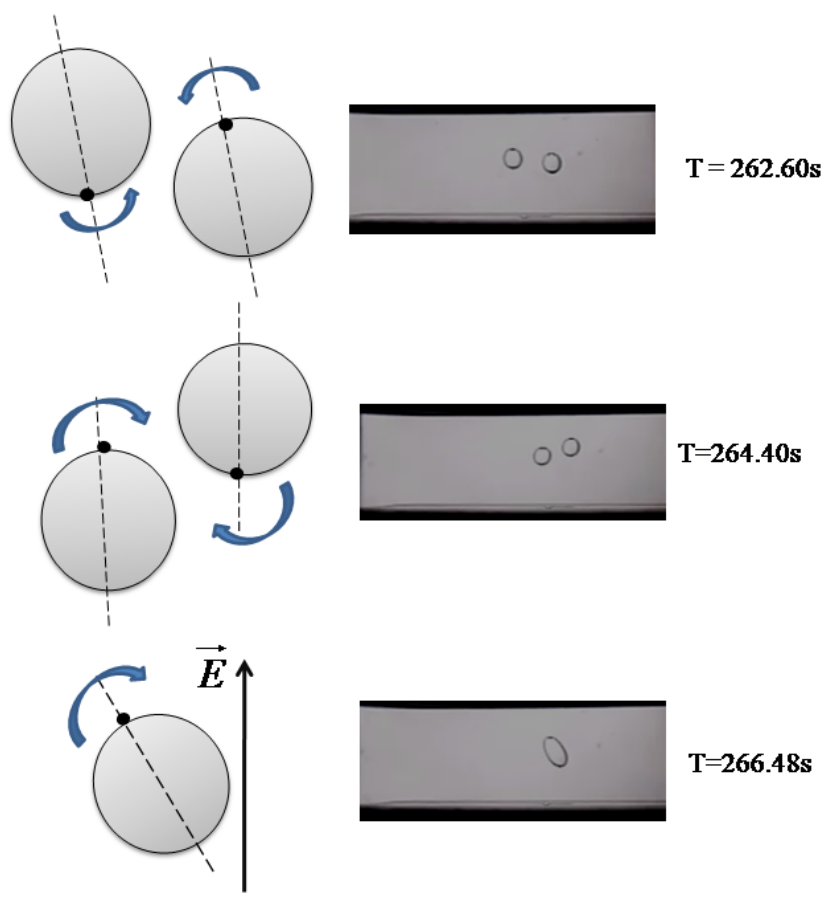

Figure 5. Schematic view of existing torque to bring drops closer together in different positions.

reverses and aligns the drops in the orientation of the electric field. However, the electric force tends to keep the drops in their previous direction. After a subtle rotation, the two drops return to their main direction, contact the two electrodes and acquire opposite charges. In every cycle, the drop-drop distance reduces due to the attractive force between opposite charges. The torque is increased and consequently the drops coalesce. The other process is after coalescence, when the two drops merge and comprise of a unique drop. This drop tends to rotate again and align itself in the electric field direction; this may occur because of the polarization that happens inside the drop which experiences a dipole moment when exposed to the electric field. This drop has a net zero charge, if neglecting the charge leakage and that equal charges are attained by the drops from the contact of the electrodes. Then the drop falls slowly due to its weight and consequently, after touching the bottom electrode, it recharges again and starts a new rhythmic motion. Similar to Fig. 2, the resultant drop fluctuates between the two electrodes, however, the drop experiences larger electrical stresses and velocity by rising the applied electric field. Therefore the deformation rate gets larger. Essentially with increasing the value of the electric field which can be defined as an voltage difference divided by the spaces of the two electrodes, the drop launches to have an acute angle at its attachment to the electrode. By increasing this value the angle increases and forms Taylor cones. In this step (Fig. 6) it can be seen higher deformation rate and a more acute angle, both at the time of contacting to the electrodes and moving between them in comparison with Fig. 2 .

\subsection{Break-up and bouncing}

Understanding the drops' breakup under the electric field plays an important role. Since the mode of breakup, determines the distribution of aqueous drops in continuous phase in addition to the size. Breakup can be considered as a technique for the emulsification and atomization in practical 


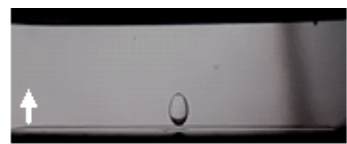

$\mathrm{T}=291.90 \mathrm{~s}$

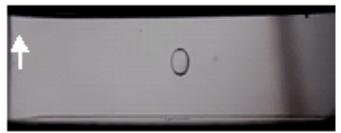

$\mathrm{T}=292.30 \mathrm{~s}$

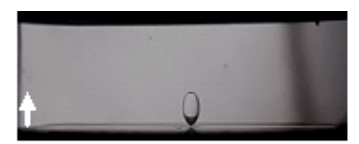

$\mathrm{T}=292.0 \mathrm{~s}$

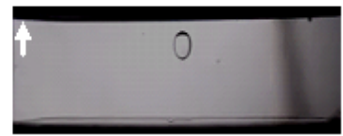

$\mathrm{T}=292.60 \mathrm{~s}$

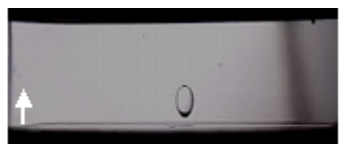

$\mathrm{T}=292.10 \mathrm{~s}$

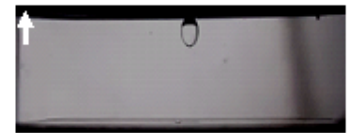

$\mathrm{T}=292.70 \mathrm{~s}$

Figure 6. Rhythmic motion for drop resulted by coalescence at $\mathrm{Ca}_{E}=0.205$.

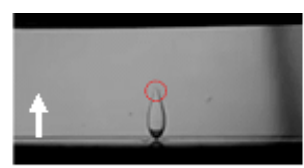

$\mathrm{T}=327.10 \mathrm{~s}$

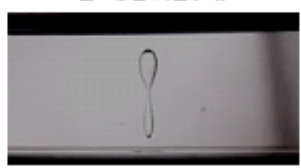

$\mathrm{T}=327.20 \mathrm{~s}$

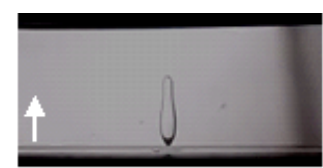

$\mathrm{T}=327.13 \mathrm{~s}$

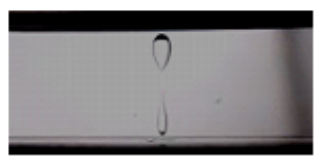

$\mathrm{T}=327.25 \mathrm{~s}$

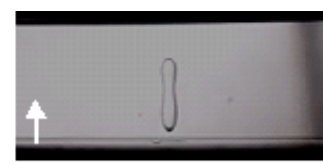

$\mathrm{T}=327.16 \mathrm{~s}$

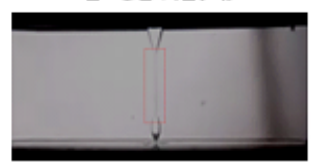

$\mathrm{T}=327.28 \mathrm{~s}$

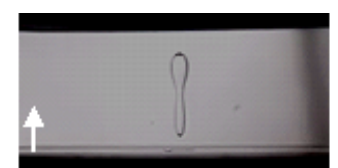

$\mathrm{T}=327.19 \mathrm{~s}$

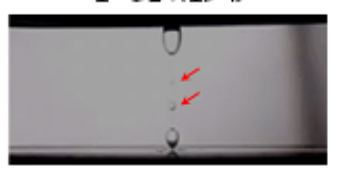

$\mathrm{T}=327.45 \mathrm{~s}$

Figure 7a. The mother drop deforms greatly into an elongated shape and divides into two daughter drops along with an expanding liquid bridge between them, which eventually breaks up into more satellite drops $\left(\mathrm{Ca}_{E}=0.216\right)$.

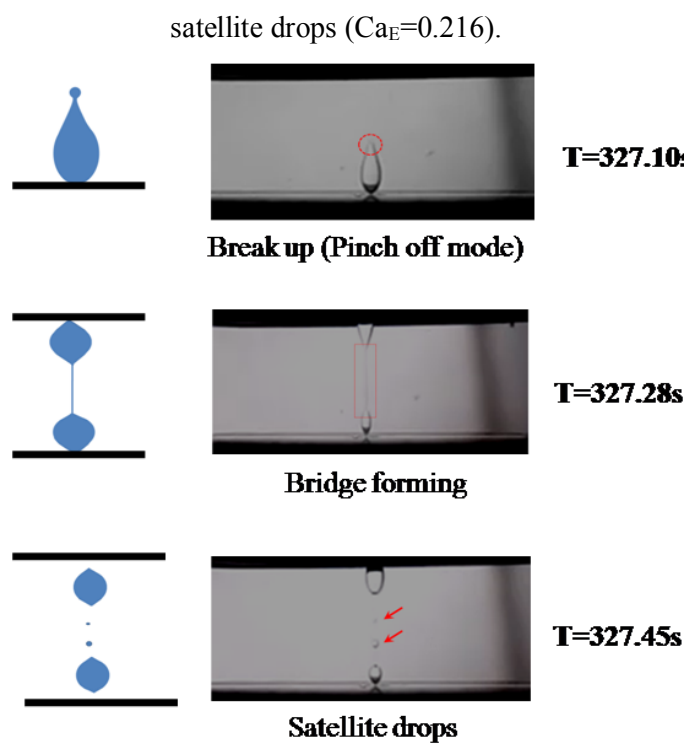

Figure $7 \mathrm{~b}$. Schematic view of breakup, creating thread and forming satellite drops.

projects as well as the enhancement of heat and mass transfer in droplet-based fluidic systems.

The equilibrium deformation, for a dispersed conductive drop moving through a dielectric medium of electrical permittivity $\varepsilon_{\mathrm{c}}$ and in the presence of applied electric field, can be obtained by balancing the electrical stresses $\left(P_{\mathrm{el}}=\varepsilon_{\mathrm{c}} E^{2}\right)$ and pressure difference at the interface of droplet. If the electrical stress overcomes the restoring capillary pressure, then the drop breaks up. Two modes of drop breakup in a strong electric field have been introduced in the literature. The first type is pinch-off where the drop forms two bulbous ends, narrowing and disintegrating at a waist. The second type is tipstreaming where the tips become sharp and tiny drops are left from its ends. Figure 7 shows the single drop that moves to the lower electrode with a $4.1 \mathrm{kV} \mathrm{cm}^{-1}$ electric field strength. Shortly after touching the electrode, a pinching region develops on the top of the drop tending to move upward towards the opposite electrode. This occurrence causes the drop to be considerably elongated while leaving the metal surface and the drop is sequentially divided into two parts connected with a liquid thread. As each of the two bulbous-end drops reach the nearby electrodes, the thread expands continuously and finally breaks up into smaller satellite droplets as a result of instability caused by capillary. The ionized aqueous droplet in silicon oil with electrical resistance lower than $10^{-5}$, is initially elongated into an ellipsoidal shape. By exceeding the, critical Weber number $(0.21$ which is in a good agreement with the numerical results of Feng and Scott (1966) and experimental results of $\mathrm{Ha}$ and Yang, 2000), the drop shape becomes unstable and varies with time. The deformation rate increases moderately until the main drop is broken into several tiny drops. Close to the ends, the deformation of the surface results in thinner drops. The pointed ends keep 


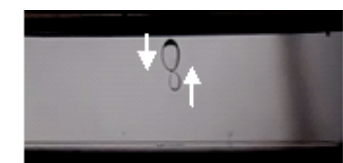

$\mathrm{T}=351.27 \mathrm{~s}$

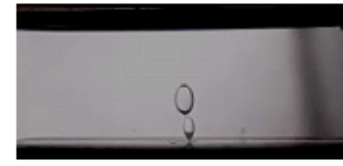

$\mathrm{T}=356.6 \mathrm{~s}$

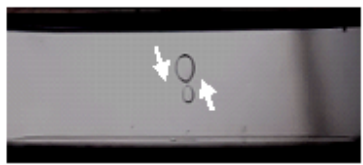

$\mathrm{T}=360.76 \mathrm{~s}$

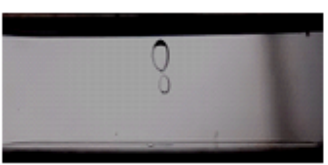

$\mathrm{T}=351.28 \mathrm{~s}$

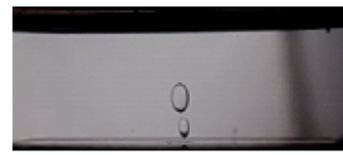

$\mathrm{T}=356.63 \mathrm{~s}$

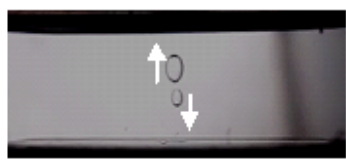

$\mathrm{T}=360.77 \mathrm{~s}$

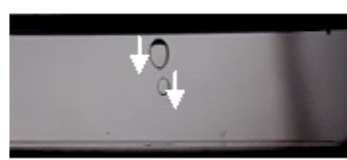

$\mathrm{T}=351.30 \mathrm{~s}$

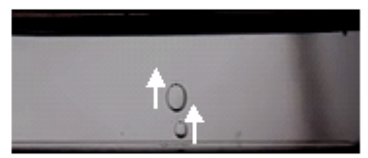

$\mathrm{T}=356.66 \mathrm{~s}$

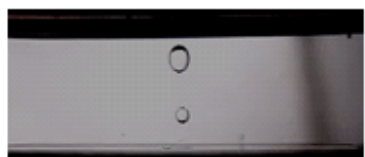

$\mathrm{T}=360.90 \mathrm{~s}$

Figure 8. Repelling phenomenon of two opposite charge drops at the top, bottom and middle positions $\left(\mathrm{Ca}_{E}=0.226\right)$.

the former trend and become slender until the ends which are now similar to bulb launch fragmentation into two distinct parts meanwhile the mother droplet changes steadily to become a thin cylindrical droplet as like as unstable liquid thread. As a result, satellite drops are produced. Initially a pinching zone is promoted (Fig. 7). Just after the forming of a pinching zone, the deformation of drop launches to increase quickly; meanwhile, the drop seeks to move upward due to its opposite charge. Therefore the drop stretches more and appears to break from the tip. It was expected that a daughter droplet is ejected from the pointed ends of the elongated drop, but as observed, the larger fraction of the stretched drop remains at the top and forms a bulbous end while the thinner part comprises the bottom of the main drop joined by a thread. At this time, the top and bottom drops reach the bottom and top electrodes respectively. The thread bursts into a few tiny droplets because of the capillary force and the thread becomes separated. These large drops experience collision with tiny drops. In this step, two phenomena occur when two or more drops exist in the domain (i.e., coalescence and repelling).

Tiny drops move based on gravity and coalesce with the bottom droplet. Therefore two main drops move between the electrodes. Traditionally, it was assumed that contacting the charged droplet with different sign are necessarily attracted each other. However if the electric field exceeds the critical value, repelling may take place rather than coalescence. As shown in Fig. 8, oppositely charged drops colloid with and repel each other. Figure 8 shows that the smaller drop, which is the consequence of the collision of tiny drops with the lower drop in the earlier step, moves upward because of the opposite charge and colloids with the larger drop that has been detached from the ground electrode. Electrical stresses operating on the charged surface causes the edge of drop to be elongated and consequently conical shape is formed. During rapid connection with the oppositely charged drop the tip recoils, gathers opposite charge and change its direction. The bottom droplet after changing its charge moves downward until it reaches the bottom electrode. The top droplet after contacting the ground electrode starts to move downward due to the Columbic force (because impact occurs near the top electrode, both drops move downward with little time difference). When the bottom droplet contacts the high voltage electrode, it senses the top droplet with a negative charge at its top point, at which point the repelling phenomenon occurs, which leads the top droplet to move upward and the bottom one to move downward. This cycle repeats and is likely to occur in the center of the domain or anywhere the two oppositely charged drops have the opportunity to contact. The main question is as follows: why do the droplets bounce? The main reason for bouncing depicted in Fig. 8, is switching charge's sign because in that way drop could gain opposite charge and reverses its former direction. As a result, in non-coalescence impact, the drop cone recoils after apparent connection with the opposite sign and changes its direction.

The repelling process continues until the value of the voltage increases to $\left(4.5 \mathrm{kV} \mathrm{cm}^{-1}\right)$. The deformation rate increases and similar to the previous part, the top drop stretches to form a dumbly shape whose greater proportion is downward while the drop that is attached to the bottom electrode tends to move upward, according to its acquired charge. Figure 9, demonstrates that after fragmenting the upper drop into two parts, the tip of the bigger-detached drops aims to move upward because of the polarization, so the tip stretches until it reaches the top electrode while the bottom end reaches the bottom small drop $(507.78 \mathrm{~s})$. Because of the high voltage strength, the second drop, which is located on the bottom electrode, generates a Taylor cone. Near the upper drop, the two cones attach and form a thread $(507.8 \mathrm{~s})$. This thread cannot endure the electric field stress and burst into many tiny drops that can coalesce together. Now there are three drops that move between the two electrodes. 

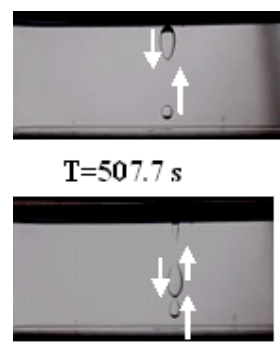

$\mathrm{T}=507.78 \mathrm{~s}$

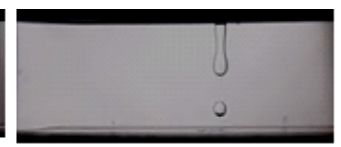

$\mathrm{T}=507.73 \mathrm{~s}$

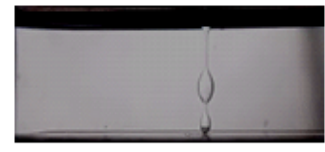

$\mathrm{T}=507.8 \mathrm{~s}$

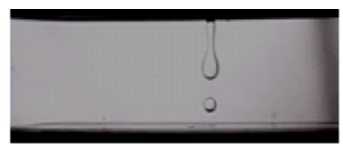

$\mathrm{T}=507.75 \mathrm{~s}$

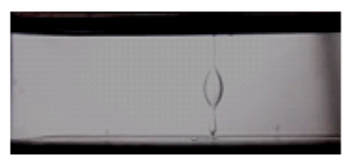

$\mathrm{T}=\mathbf{5 0 7 . 8 3 \mathrm { s }}$

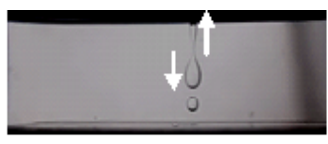

$\mathrm{T}=\mathbf{5 0 7 . 7 6 \mathrm { s }}$

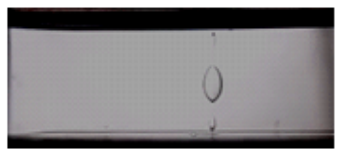

$\mathrm{T}=507.96 \mathrm{~s}$

Figure 9. Making a thread due to large elongation and increasing the voltage $\left(\mathrm{Ca}_{E}=0.26\right)$.

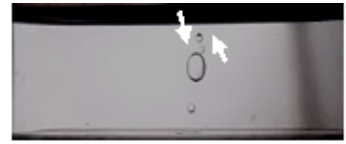

$\mathrm{T}=546.0 \mathrm{~s}$

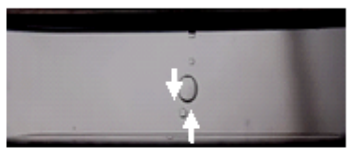

$\mathrm{T}=548.1 \mathrm{~s}$

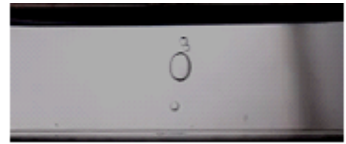

$\mathrm{T}=546.1 \mathrm{~s}$

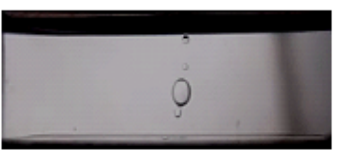

$\mathrm{T}=548.2 \mathrm{~s}$

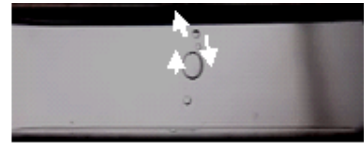

$\mathrm{T}=546.2 \mathrm{~s}$

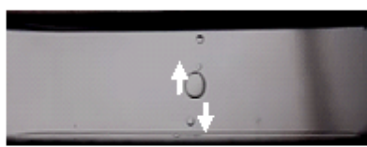

$\mathrm{T}=548.3 \mathrm{~s}$

Figure 10. Bouncing droplets $\left(\mathrm{Ca}_{E}=0.26\right)$.

\subsection{Multi-drops motion, coalescence, repulsion and break up}

By increasing the voltage, the bigger drop stretches considerably and in a certain moment, detaches a small drop from its tip. These four drops with different sizes are colliding and showing non-coalescence behavior, and during each contact they conduct, charge to each other and reverse their directions, as shown in Fig. 10.

Non-coalesce behavior is not the only feature that occurs in a high voltage electric field. In addition to the explanation stated by Ristenpart et al. (2009) which described the reasons to distinguish coalescence from repelling, and considering the presence of multiple droplets, the possibility of coalescence occurring during impact is not far-fetched. However before supporting the former argument, it is worth mentioning the reasons for repelling as fully described by Ristenpart et al. (2009). As mentioned by Khorshidi et al. (2010), the threshold field strength for droplet, hinges strongly on the amount of charges which has been transmitted during connection with the former interface. Because the charge comprises ions in the water droplet, these ions during bouncing process could transfer from one droplet to another one. Some mechanisms are suggested for charge transmitting such as conduction across the continuous phase (oil), breakdown of dielectric medium or smaller drops are sprayed by electric force (electro spray) however in the case of silicon oil, because of the aqueous thread which is formed during connection, the possibility of conduction of ions is presumably stronger than other reasons. The observation indicates that the drop takes double-cone shape near the bridge. The capillary pressure inside the thread is approximated by the YoungLaplace equation as follows:, $(\cot \theta-1) \gamma / r_{m}$ while the angle is the key factor which determines the fluid flow. It means that the drops with sharper cones generate the higher pressure difference in the bridge and consequently leads the drop to be bounced. If the voltage is high enough, to break the dielectric medium (oil), then the drops prior to contact could be neutralized and the surface tension results in recoil. In other case joule heating is assumable. During connection process, equilibration of charges may heat the liquid in the thread zone and affect the neck by Maragoni force or vaporization and conductivity in this case is a leading factor. The capillary waves at connection time, expands liquid thread between them. Larger positive curvature in contrast with negative one leads to higher positive pressure in the neck than inside the droplet and breakup is developed. In other side, larger negative curvature generates higher pressure inside the droplet than in the neck and fluid is derived in to the thread and eventually coalescence occurs. During this motion, multiple drops contact each other, and the chance of coalescence and repelling happening is unavoidable. However, repelling typically occurs between them. By increasing the electric field, there is an intention to form an elongated shape again. Previously two cases are studied where the drop is placed on the top and bottom electrodes. However this is not the only moment when the drop breaks. Typically the drop can break when moving through the gap between the electrodes $(T=629.513 \mathrm{~s})$. In Fig. 11, the top section of the drop stretches and reaches the top electrode while the bottom 


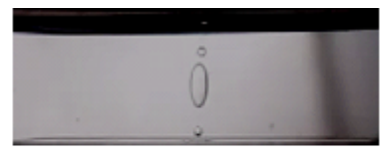

$\mathrm{T}=625.1 \mathrm{~s}$

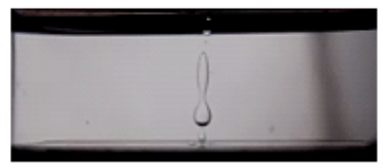

$\mathrm{T}=629.52 \mathrm{~s}$

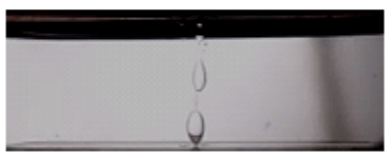

$\mathrm{T}=638.9 \mathrm{~s}$

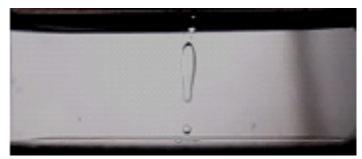

$\mathrm{T}=629.513 \mathrm{~s}$

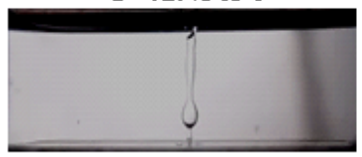

$\mathrm{T}=629.55 \mathrm{~s}$

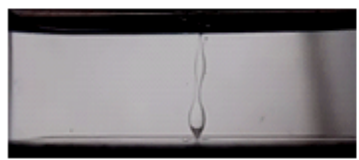

$\mathrm{T}=639.0 \mathrm{~s}$

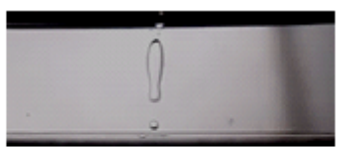

$\mathrm{T}=629.516 \mathrm{~s}$

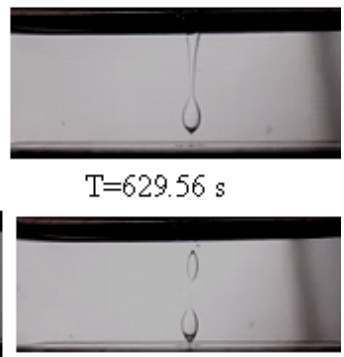

$\mathrm{T}=639.08 \mathrm{~s}$

Figure 11. Pinch-off and forming thread $\left(\mathrm{Ca}_{E}=0.321\right)$.

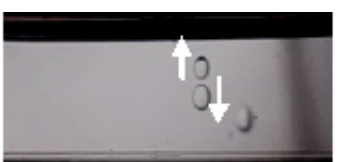

$\mathrm{T}=773.70 \mathrm{~s}(1)$

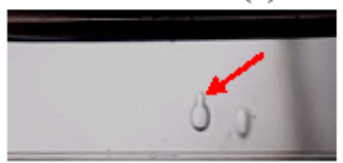

$\mathrm{T}=773.94 \mathrm{~s}$

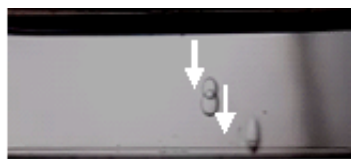

$\mathrm{T}=773.80 \mathrm{~s}(2)$

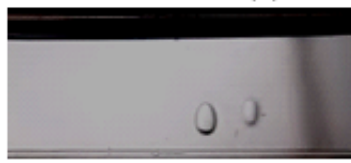

$\mathrm{T}=774.01 \mathrm{~s}$
Figure 12. The second type of droplet coalescence $\left(\mathrm{Ca}_{E}=0.334\right)$.

part reaches the bottom electrode. The thread appears, bursts and multiple drops form burst. This is a conventional cycle that repeats itself. These tiny drops can also generate a chain between the main drops and form a thread and short contact between the electrodes. The pinch-off appears to be a dominant mode of break up. The sizes of the main droplets and satellite drops become sensitive to the strength of electric field. An increase in the electric potential leads to a narrower size distribution of both droplets and satellites, and it eventually becomes impossible to distinguish drops from satellites.

As mentioned earlier, three phenomena can occur; contemporary break up, coalescence and repelling. Many studies revealed that coalescence happens at low strength of applied electric field. By increasing the value, the contact angle is sufficiently sharp to permit the bridge' pressure to be greater than the drops' pressure and repelling therefore occurs. As the results show ( $T=773.8 \mathrm{~s})$, the side-by side coalescence is expected in three-dimensional behaviors. It is obvious that according to the nature of the random movement of drops, there may be an instance that one drop gives a negative charge as the result of the short contact and wants to move for example upward (positive charge). The drop which transferred its opposite positive charge to its neighbor drop

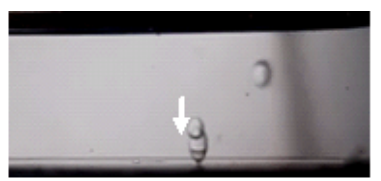

$\mathrm{T}=786.02 \mathrm{~s}(1)$

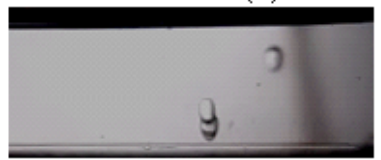

$\mathrm{T}=786.08 \mathrm{~s}$

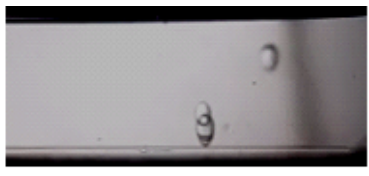

$\mathrm{T}=786.05 \mathrm{~s}(2)$

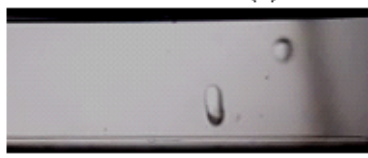

$\mathrm{T}=786.12 \mathrm{~s}$
Figure 13. The first type of droplet coalescence $\left(\mathrm{Ca}_{E}=0.360\right)$.

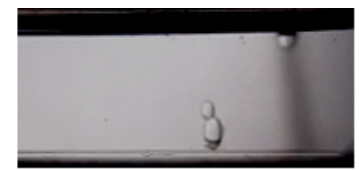

$\mathrm{T}=796.53 \mathrm{~s}$

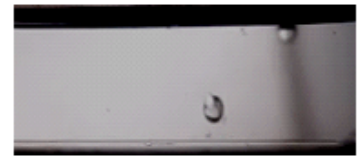

$\mathrm{T}=796.65 \mathrm{~s}$

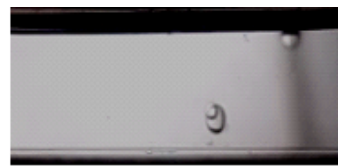

$\mathrm{T}=796.57 \mathrm{~s}$

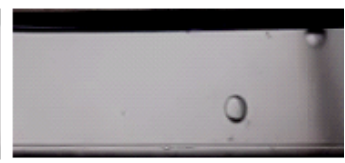

$\mathrm{T}=796.68 \mathrm{~s}$
Figure 14. The first type of droplet coalescence $\left(\mathrm{Ca}_{E}=0.374\right)$.

receives minus charge from it through the bridge (Fig. 12). The drop that attained the positive charge moves upward, contacts the ground electrode and acquires a negative charge. Because of the electrostatic force and gravity the drop moves downward. Coalescence occurs when the first drop has not yet reached the high voltage and meets the other drop.

Figure 13 shows two similarly (negative) charged drops come into contact after several prior repulsions, however they do not coalesce until the lower drop touches the metal surface, attains a positive charge and merges with the oppositely 


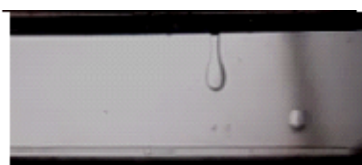

$\mathrm{T}=798.12 \mathrm{~s}$

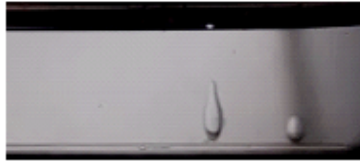

$\mathrm{I} \cdot=798.33 \mathrm{~s}$

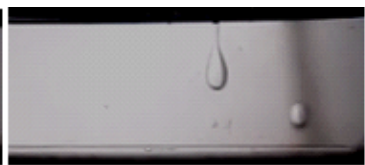

$\mathrm{T}=798.13 \mathrm{~s}$

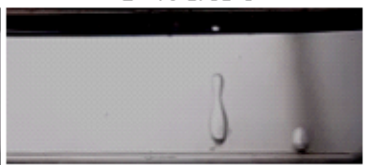

$\mathrm{T}=798.36 \mathrm{~s}$

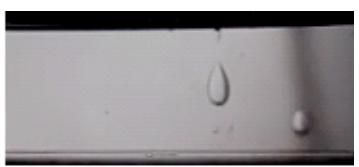

$\mathrm{T}=798.15 \mathrm{~s}$

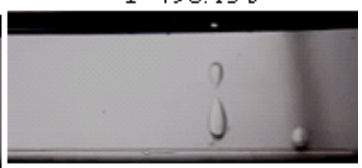

$\mathrm{T}-798.38$

Figure 15. Pinch-off at top and bottom electrode $\left(\mathrm{Ca}_{E}=0.388\right)$.
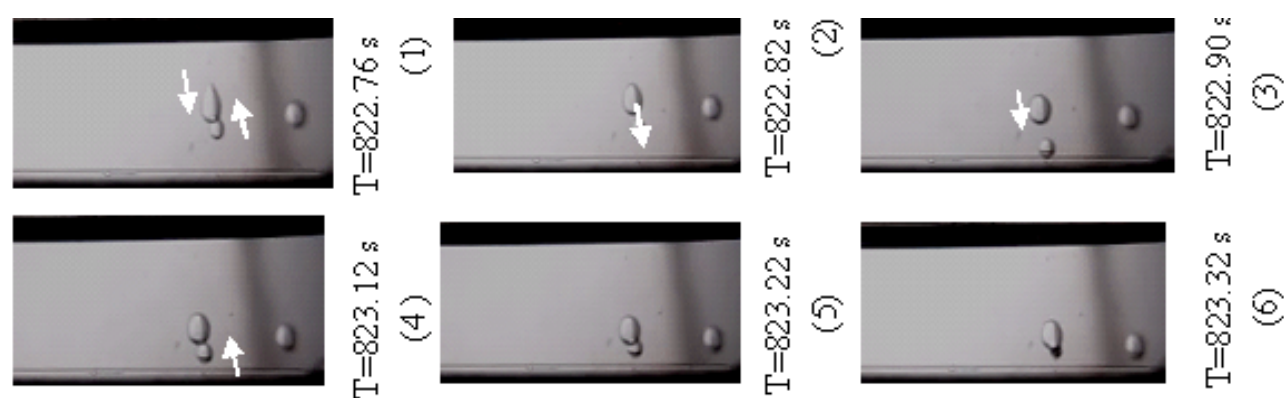

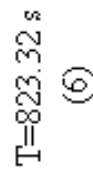

Figure 16. The third type of droplet coalescence $\left(\mathrm{Ca}_{E}=0.388\right)$.

charged neighbor drop. Here, coalescence has been detected for two oppositely charged drops.

In Fig. 14 coalescence occurs again. This time, the bigger droplet attaches at the high voltage, acquires positive charge and coalesces with its upper droplet which has a negative charge. According to previous studies in the high voltage field, repelling is dominant, here, however, it seems that the direction (contact angle) at the time of contact plays an important role and leads to coalescence. The connection's line is not straight and the contact occurs side to side. As discussed above, the orientation of the drops play an important role in investigating whether coalescence or bouncing is likely to happen.

Increasing the electric field causes break up (pinch-off) to occur simultaneously. The first instance occurs, when the drop contacts the top electrode and then the bottom electrode (Fig. 15).

As mentioned above, it is possible to have coalescence in a high electric field. In Fig. 16, the top drop becomes close to the bottom one. There are two options available; coalescence and repelling. Because two opposite charges attract, it is expected that they merge and make a bigger drop, however, this has not happened and repelling has occurred instead of coalescence. So based on Ristenpart et al. (2009) charge transmission has occurred and the bottom drop obtained the negative charge and started moving downward. Because of this charge changing, it is assumed that the top drop would acquire positive charge and move upward, but the next frame does not show this movement. In contrast, it tends to fall with its weight and the vertical distance from the bottom electrode decreases. After acquiring a positive charge from the bottom electrode it starts moving upward and contacts with the upper drop which is resting in its former position or has moved a short distance, and this is the time at which coalescence occurs. If it is justifiable that coalescence occurs with two opposite charges there would be one option left, i.e., the average amount of charge for the upper drop is negative. Therefore it may be correct to presume that the upper drop does not share its entire charge with the small droplet and that the amount of transmitted charge is not sufficient to create a positive charge within the upper droplet. This case is considered the third type of coalescence. If the last option is true, then acceleration towards the high voltage is expected for the top droplet based on electrophoretic force; however the top droplet moves very slowly similar to the droplet with zero charge. The question remains regarding the charge transmission.

\section{Conclusions}

In the current study, the deformation, electrical charging, coalescence and breakup of water drops moving vertically between two horizontal electrodes in an insulating liquid were investigated experimentally. Any changes that occurred during rhythmic motion have been addressed. The study 
confirms the recent results obtained by researchers, and the main observations are listed as follows:

- Increasing the voltage results in a Taylor cone when droplets are resting on or reaching the electrodes. By adding the voltage, the angles become smaller and the droplets leave the electrodes. The deformation rate increases with the applied voltage.

- The torque, which exists at the points of the drops, causes rotation while the electric field wants to align them in the vertical direction. This interaction continues until the distance is short enough to cause coalescence. Additionally coalescence does not necessarily occur from the tip and side by side coalescence is occasionally expected.

- After coalescence, assuming the charge transmission occurs equally between the oppositely charged droplets, the consequent droplet, which has a bigger diameter, rotates such that it aligns itself in the electric field direction (because of the polarization). Because of zero charge, the droplet falls with its weight.

- At high voltage, because of the polarization, the droplet elongates and finally disintegrates into two or more tiny droplets. These tiny droplets either coalesce or bounce against each other. Bouncing occurs because of the changing charges.

- When a thread is formed between electrodes it disintegrates immediately.

- There are three types of coalescence modes mentioned above: (i) typical coalescence, which is between oppositely charged droplets, (ii) coalescence between two droplets with the same sign maybe because of hydrodynamic coalescence and (iii) coalescence after bouncing where one of the droplets in the transmission section does not move to the opposite electrode but moves very slowly and relatively rests at its first position (the time of contact) to coalesce with the second droplet (Fig. 16).

- At very high voltage, droplets gather in a certain line, which is not in an electric field direction and deviates from the vertical line. The chain-like shape allows currents to pass through the distance between the electrodes, a spark is therefore inevitable.

Acknowledgements. We would like to express our deep appreciation to B. Khorshidi (university of Alberta) for his valuable comments during the preparation of the work.

Edited by: A. Barari

Reviewed by: four anonymous referees

\section{References}

Ahn, K., Agresti, J., Chong, H., Marquez, M., and Weitz, D. A.: Electrocoalescence of drops synchronized by size-dependent flow in microfluidic channels, Appl. Phys. Lett., 88, 264105, doi:10.1063/1.2218058, 2006.

Aldaeus, F., Lin, Y., Amberg, G., and Roeraade, J.: Multi-step dielectrophoresis for separation of particles, J. Chromatogr. A, 1131, 261-266, 2006

Allan, R. S. and Mason, S. G.: Particle behavior in shear and electric fields; I:deformation and burst of fluid drops, Proc. Roy. Soc. Lond. A, 267, 45-61, 1962.

Bailey, A. G.: Electrostatic spraying of liquids, Wiley, New York, 1988, dynamic processes in electrospraying, J. Aerosol Sci., 30 (Suppl. 1), 549-550, 1999.

Baygents, J. C. and Saville, D. A.: The circulation produced in a drop by an electric field: a high field strength electrokinetic model, in: Drops and Bubbles: Third Int. Colloq., edited by: Wang, T. G., American Institute of Physics, 7-17, 1989.

Chiesa, M., Melheim, J. A., Pedersen, A., Ingebrigtsen, S., and Berg, G.: Forces acting on water droplets falling in oil under the influence of an electric field: numerical predictions versus experimental observations, Eur. J. Mech. B-Fluid., 24, 717-732, 2005.

Dong, W., Li, R. Y., Yu, H. L., and Yan, Y. Y.: An investigation of behaviors of a single bubble in a uniform electric field, Exp. Therm. Fluid Sci., 30, 579-586, 2006.

Eow, J. S. and Ghadiri, M.: Motion, deformation and break-up of aqueous drops in oils under high electric field strengths, Chem. Eng. Process., 42, 259-272, 2003a.

Eow, J. S. and Ghadiri, M.: Drop-Drop coalescence in an electric field: the effects of applied electric field and electrode geometry, Colloid. Surface. A, 219, 253-279, 2003b.

Eow, J. S., Ghadiri, M., Sharif, A. O., and Williams, T. J.: Electrostatic enhancement of coalescence of water droplets in oil: a review of the current understanding, Chem. Eng. J., 84, 173-192, 2001

Feng, J. Q. and Scott, T. C.: A computational analysis of electrohydrodynamics of a leaky dielectric drop in an electric field, J. Fluid Mech., 311, 289-326, 1966.

Ha, J. W. and Yang, S. M.: Deformation and break-up of Newtonian and non-newtonian conducting drops in an electric field, J. Fluid Mech., 405, 131-156, 2000.

Hase, M., Watanabe, S. N., and Yoshikawa, K.: Rhythmic motion of a droplet under a dc electric field, Phy. Rev. E., 74, 046301, doi:10.1103/PhysRevE.74.046301, 2006.

Hokmabad, B. V., Sadri, B., Charan, M. R., and Esmaeilzadeh, E. An experimental investigation on hydrodynamics of charged water droplets in dielectric liquid medium in the presence of electric field, Colloid. Surface. A, 401, 17-28, 2012.

Khorshidi, B., Jalaal, M., Esmaeilzadeh, E., and Mohammadi, F. Characteristics of deformation and electrical charging of large water drops immersed in an insulating liquid on electrode surface, Colloid Interface Sci., 352, 211-220, 2010.

Pikal, M. J.: The role of electroosmotic flow in transdermal iontophoresis, Advance Drug Delivery, Reviews, 46, 281-305, 2001.

Ristenpart, W. D., Bird, J. C., Belmonte, A., Dollar, F., and Stone, H. A.: Non-coalescence of oppositely charged drops, Nature, 461 , 377-380, 2009. 
Sherwood, J. D.: Breakup of fluid droplets in electric and magnetic fields, J. Fluid Mech., 188, 133-146, 1988.

Taylor, G. L.: Proc. Studies in electrohydrodynamics; I: the circulation produced in a drop by an electric field, Roy. Soc. A, 291, 159-167, 1966.

Torza, S., Cox, R. G., and Mason, S. G.: Electrohydrodynamic deformation and burst of liquid drops, Philos. Trans. R. Soc. Lond. A, 269, 295-319, 1971.
Vizika, O. and Saville, D. A.: The electrohydrodynamic deformation of drops suspended in liquids in steady and oscillatory electric fields, J. Fluid Mech., 239, 1-21, 1992. 